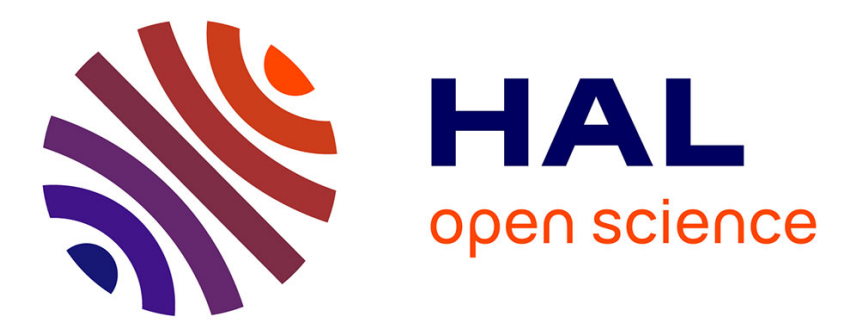

\title{
Equations of energy exchanges in variable density turbulent flows
}

Dorian Dupuy, Adrien Toutant, Françoise Bataille

\section{To cite this version:}

Dorian Dupuy, Adrien Toutant, Françoise Bataille. Equations of energy exchanges in variable density turbulent flows. Physics Letters A, 2018, 382 (5), pp.327-333. 10.1016/j.physleta.2017.11.026 . hal01867338

\section{HAL Id: hal-01867338 \\ https://hal.science/hal-01867338}

Submitted on 4 Sep 2018

HAL is a multi-disciplinary open access archive for the deposit and dissemination of scientific research documents, whether they are published or not. The documents may come from teaching and research institutions in France or abroad, or from public or private research centers.
L'archive ouverte pluridisciplinaire HAL, est destinée au dépôt et à la diffusion de documents scientifiques de niveau recherche, publiés ou non, émanant des établissements d'enseignement et de recherche français ou étrangers, des laboratoires publics ou privés. 


\title{
Equations of energy exchanges in variable density turbulent flows
}

\author{
Dorian Dupuy ${ }^{1}$, Adrien Toutant*1, and Françoise Bataille ${ }^{1}$ \\ ${ }^{1}$ PROMES CNRS, Université de Perpignan Via Domitia, Rambla de la \\ thermodynamique, Tecnosud, 66100 Perpignan, France
}

(Dated: 23 October 2017)

\begin{abstract}
This paper establishes a new formulation of the energy exchanges between the different parts of total energy. The decomposition uses the Reynolds averaging. This leads to a ternary decomposition of kinetic energy into the turbulence kinetic energy, the mean kinetic energy and the mixed kinetic energy, acting as an exchange term between the mean and turbulent motion. The formulation is then extended to distinguish a mean and fluctuating density part of each part of total energy. The formulation thus includes the mean density turbulence kinetic energy, product of the mean density and the half-trace of the velocity fluctuation correlation tensor. Its evolution equation is given in the spectral domain.
\end{abstract}

\section{Introduction}

This paper addresses the energy exchanges in turbulent flows with highly variable fluid properties. This covers flows with a high Mach number (high speed flows), such as the flows around a high-speed aircraft, or through a high speed jet or a nozzle [25], and low Mach number flows submitted to a strong temperature gradient, found for instance in heat exchangers, propulsion systems or nuclear or concentrated solar power plants $[4,37,17,45,46,47,49]$. The study of the energy exchanges between the different parts of total energy is a useful tool for both turbulence modelling and the fundamental understanding of turbulence. More detailed information is obtained through the study of the energy exchanges in the spectral domain $[34,18,19,53,54$, $19,36,20,7,11,12,13,14,31,38]$. However, while kinetic energy is fundamental property of any flow, it is not the case of its decomposition into turbulence kinetic energy and mean kinetic energy.

In incompressible flows with constant fluid properties, such decomposition is unique. The averaged kinetic energy is decomposed clearly, unambiguously and straightforwardly into the sum of two contributions: the kinetic energy of the mean

\footnotetext{
*Corresponding author : adrien.toutant@univ-perp.fr
} 
motion associated with the mean velocity and the kinetic energy of the turbulent motion associated with the velocity fluctuation [see e.g. 51, 42]. In compressible flows with highly variable density, this analysis is hindered by additional density-velocity correlations. The decomposition of kinetic energy becomes more complex and arbitrary. It is even more difficult in the spectral domain. The choice ultimately depends on the physical role given to the additional density-velocity correlations with respect to what constitutes the mean motion and the turbulent motion [10]. The most popular and successful decomposition extends the incompressible decomposition to the compressible case through the introduction of a density weighted averaging. This decomposition was widely developed by Favre [21, 22, 23]. Since, it has been used extensively by various authors [32, 29, 26, 35, 41]. Another approach, the mixed weighted decomposition, mixes density weighted averaging and Reynolds averaging. It was first introduced by Bauer et al. [6] and further studied by Ha Minh et al. [27, 28]. In this formulation, kinetic energy is seen as the product of the velocity and the density weighted velocity. In a third method, kinetic energy is decomposed using a change of variable based on the density square root weighted velocity. This decomposition was first proposed by Yih [52] then adopted by various authors $[30,44,15,1,2]$. This change of variable allows the study of kinetic energy to be extended easily to the spectral domain. Finally, Chassaing [8] [see also 16, 3, 9, 10] suggested the decomposition of kinetic energy using the Reynolds averaging. From a modelling perspective, the use of the unweighted averaging may be advantageous in low Mach number flows, in which the energy conservation acts as a constraint on the divergence of the velocity [40]. The square of the fluctuating velocity (without the density) is also encountered for instance in the modelling of two-phase flows [50] or in variable density flows, provided the momentum equation is divided by the density before averaging [48]. In a variable density setting, the use of the Reynolds averaging necessarily leads to the decomposition of kinetic energy into three parts, called ternary decomposition. The kinetic energy is thus split into turbulence kinetic energy, mean kinetic energy and a mixed kinetic energy, related to both the mean and turbulent motions. However, we believe the underlying idea behind the ternary decomposition has not been taken to its logical conclusion as no interaction between the mixed kinetic energy and another part of total energy was identified.

This paper aims to establish a new formulation of the energy exchanges between the different parts of total energy in a ternary decomposition that gives to the mixed kinetic energy a full role. The formulation is compared to the formulation of Chassaing [8] and the differences between the two formulations with regard to the physical interpretation of the terms are discussed. We then take the decomposition further and split the density into a mean and fluctuating part. This leads to the definition of the mean density part of total energy and the fluctuating density part of total energy. The mean density turbulence kinetic energy, product of the mean density and the half-trace of the velocity fluctuation correlation tensor, appears in the mean density part of the decomposition as exchanging energy with the other parts of total energy. This quantity is approximately equal to the turbulence kinetic energy in flows satisfying Morkovin's hypothesis [39]. 
Once the new formulation of the energy exchanges established, we focus more specifically on the mean density turbulence kinetic energy. We establish its evolution equation in spectral domain, recognizing that the mean density turbulence kinetic energy has with the Reynolds averaging a clear spectral equivalent. The spectral equation extends the spatial equation to the spectral domain, associating to each spatial term a spectral equivalent. To the knowledge of the authors, this has not been achieved in the literature for variable density flows. A purely spectral term that redistributes the energy between scales is identified, as in the work of Lee and Moser [31] and Mizuno [38]. In order to carry out the Fourier transform, we consider a flow with two homogeneous and periodic directions. This does not lead to a loss of generality as the equations given may easily be adapted to a flow with one or three homogeneous directions.

The complete representation of the energy exchanges between the different parts of total energy is presented in section 2 and the equation of the mean density turbulence kinetic energy in the spectral domain in section 3.

\section{Energy exchanges between the different parts of total energy in the ternary decomposition}

\subsection{General considerations}

In this section, we describe a new formulation of the energy exchanges between the different parts of total energy in a ternary decomposition. We will establish the formulation obtained from the decomposition of velocity, but not density, with the Reynolds averaging, referred to as the one-stage formulation in this paper, and from the decomposition of both the velocity and density, referred to as the twostage formulation in this paper. The two-stage formulation is required to write the spectral equation of the mean density turbulence kinetic energy. We first define here a few useful quantities and give some general remarks on the derivation of the formulation.

The total energy per unit volume $\rho(E+I)$ is a conservative quantity. Its components however are not as they exchange energy among themselves. In the following, the evolution equation of each part of total energy in the ternary representation will be given and we will identify the energy exchanges between these quantities. Many consistent formulations of the energy exchanges can be proposed. The formulation was devised according to the following criteria:

- Each term of the formulation must be either interpreted as a conservative energy transfer or an interaction with exactly one of the other parts of total energy.

- If a term is to be interpreted as a conservative energy transfer, it must be written in a conservative form, that is as a divergence; otherwise, it must be written in a non-conservative form. 
- The formulation must be symmetrical, in particular with respect to the manner in which it deals with fluctuations and statistically averaged quantities.

- The formulation must correctly behave when considering a limit case such as laminar, homogeneous or incompressible flows. In particular, a quantity that becomes instantaneously equal to zero must not be associated with any energy exchange.

We consider a non-relativistic compressible flow with highly variable fluid properties under the continuity hypothesis. Without loss of generality, no body forces are taken into account which means gravity is neglected and there is no heat source. The flow is governed by the Navier-Stokes equations under the following form [24]:

- mass conservation

$$
\frac{\partial \rho}{\partial t}+\frac{\partial \rho U_{j}}{\partial x_{j}}=0
$$

- momentum conservation

$$
\frac{\partial \rho U_{i}}{\partial t}+\frac{\partial \rho U_{j} U_{i}}{\partial x_{j}}=\frac{\partial \Upsilon_{i j}}{\partial x_{j}}
$$

- energy conservation

$$
\frac{\partial \rho I}{\partial t}+\frac{\partial \rho U_{j} I}{\partial x_{j}}=\frac{\partial}{\partial x_{j}}\left(\lambda \frac{\partial T}{\partial x_{j}}\right)+\Upsilon_{i j} \frac{\partial U_{i}}{\partial x_{j}}
$$

with $\rho$ the density, $T$ the temperature, $I$ the internal energy per unit mass, $t$ the time, $U_{i}$ the $i$-th component of the velocity, $\Upsilon_{i j}$ the component of the total stress tensor with the $i$ and $j$ indices and $x_{i}$ the Cartesian coordinate in the $i$-th direction. Einstein summation convention is used. The total stress tensor $\Upsilon_{i j}$ is given by the contributions of the viscous shear stress tensor and of the pressure stress. We will keep the total stress tensor undissociated throughout this paper because the pressure and viscous contributions are formally similar.

\subsection{One-stage formulation}

The instantaneous total energy per unit volume $\rho(E+I)$ is the sum of the instantaneous kinetic energy per unit volume $\rho E$ and the internal energy per unit volume $\rho I$. In the ternary decomposition, the kinetic energy is decomposed into three parts by splitting the velocity into a mean and fluctuating part [following 43], namely $U_{i}=\bar{U}_{i}+u_{i}^{\prime}$, where the overline $(\overline{ })$ denotes the statistical average and the prime symbol $\left({ }^{\prime}\right)$ the fluctuating part. We use a lowercase $u^{\prime}$ for the velocity fluctuation for a better visual differentiation but there is no further underlying differences. We obtain [8]

$$
\rho E=\frac{1}{2} \rho U_{i} U_{i}=\rho \underline{E}+\rho e+\rho \underline{e}
$$


with $\rho \underline{E}=\frac{1}{2} \rho \bar{U}_{i} \bar{U}_{i}$ the mean kinetic energy, associated with the mean motion, $\rho e=\frac{1}{2} \rho u_{i}^{\prime} u_{i}^{\prime}$ the turbulence kinetic energy, associated with the turbulent motion, and $\rho \underline{e}=\rho u_{i}^{\prime} \bar{U}_{i}$ the mixed kinetic energy, associated with both the mean and turbulent motion. This results in a fourfold decomposition of total energy.

The evolution equation of total energy $\overline{\rho(E+I)}$ is given by

$$
\frac{\partial \overline{\rho(E+I)}}{\partial t}=\overline{\Phi^{c}}+\overline{\Phi^{\Upsilon}}+\overline{\Phi^{\lambda}}
$$

with: $\Phi^{c}$ the convection, $\Phi^{\Upsilon}$ the transfer by the total stress and $\Phi^{\lambda}$ the transfer by conduction, given by:

$$
\begin{aligned}
\Phi^{c} & =-\frac{\partial \rho U_{j}(E+I)}{\partial x_{j}}, \\
\Phi^{\Upsilon} & =\frac{\partial \Upsilon_{i j} U_{i}}{\partial x_{j}}, \\
\Phi^{\lambda} & =\frac{\partial}{\partial x_{j}}\left(\lambda \frac{\partial T}{\partial x_{j}}\right) .
\end{aligned}
$$

These three terms are conservative terms that represent the transfer of total energy by three different physical phenomena. With the fourfold decomposition of total energy, this equation becomes four equations associated with each part of total energy. This has two effects. First, the various conservative energy transfer terms are distributed among the four parts of kinetic energy. Secondly, additional nonconservative terms emerge. From the decomposition of total energy into kinetic energy and internal energy appears a new term $\mathcal{E}$ that represents the interaction between these two quantities. From the decomposition of kinetic energy into three terms appears two new terms $\underline{\mathcal{P}}$ and $\mathcal{P}$ that represent an interaction between the different parts of kinetic energy.

The energy exchanges between the four parts of total kinetic energy may be written as:

$$
\begin{aligned}
& \frac{\partial \overline{\rho \underline{E}}}{\partial t}=\overline{\Phi^{c}}+\overline{\Phi^{\Upsilon}}+\underline{\overline{\mathcal{P}}}+\overline{\mathcal{E}}, \\
& \frac{\partial \overline{\rho e}}{\partial t}=\overline{\varphi^{c}}+\overline{\varphi^{\Upsilon}}+\overline{\mathcal{P}}+\bar{\epsilon}, \\
& \frac{\partial \bar{\rho} \underline{\underline{e}}}{\partial t}=\overline{\varphi^{c}}-\overline{\mathcal{P}}-\underline{\overline{\mathcal{P}}}, \\
& \frac{\partial \overline{\rho I}}{\partial t}=\overline{\Phi^{T, c}}+\overline{\Phi^{\lambda}}-\overline{\overline{\mathcal{E}}}-\bar{\epsilon},
\end{aligned}
$$

where we identify the following terms:

- the convection $\Phi^{c}$, decomposed into: $\Phi^{c}$ associated with the mean kinetic energy, $\varphi^{c}$ associated with the turbulence kinetic energy, $\underline{\varphi}^{c}$ associated with the mixed kinetic energy and $\Phi^{T, c}$ associated with internal energy,

$$
\underline{\Phi}^{c}=-\frac{\partial \rho U_{j} \underline{E}}{\partial x_{j}}
$$




$$
\begin{aligned}
\varphi^{c} & =-\frac{\partial \rho U_{j} e}{\partial x_{j}}, \\
\underline{\varphi}^{c} & =-\frac{\partial \rho U_{j} \underline{e}}{\partial x_{j}}, \\
\Phi^{T, c} & =-\frac{\partial \rho U_{j} I}{\partial x_{j}},
\end{aligned}
$$

- the transfer by the total stress $\Phi^{\Upsilon}$, decomposed into: $\underline{\Phi}^{\Upsilon}$ associated with the mean kinetic energy and $\varphi^{\Upsilon}$ associated with the turbulence kinetic energy,

$$
\begin{gathered}
\underline{\Phi}^{\Upsilon}=\frac{\partial \Upsilon_{i j} \bar{U}_{i}}{\partial x_{j}}, \\
\varphi^{\Upsilon}=\frac{\partial \Upsilon_{i j} u_{i}^{\prime}}{\partial x_{j}},
\end{gathered}
$$

- the interaction between the turbulence kinetic energy and the mixed kinetic energy $\mathcal{P}$,

$$
\mathcal{P}=-\rho u_{i}^{\prime} U_{j} \frac{\partial \bar{U}_{i}}{\partial x_{j}}+\rho u_{i}^{\prime} \overline{U_{j} \frac{\partial U_{i}}{\partial x_{j}}}-\rho u_{i}^{\prime} \frac{\overline{1} \frac{\partial \Upsilon_{i j}}{\partial x_{j}}}{,}
$$

- the interaction between the mean kinetic energy and the mixed kinetic en$\operatorname{ergy} \underline{\mathcal{P}}$,

$$
\underline{\mathcal{P}}=-\rho \bar{U}_{i} U_{j} \frac{\partial u_{i}^{\prime}}{\partial x_{j}}+\rho \bar{U}_{i}\left(U_{j} \frac{\partial U_{i}}{\partial x_{j}}\right)^{\prime}-\rho \bar{U}_{i}\left(\frac{1}{\rho} \frac{\partial \Upsilon_{i j}}{\partial x_{j}}\right)^{\prime}
$$

- the interaction between kinetic energy and internal energy $\mathcal{E}$, decomposed into: $\underline{\mathcal{E}}$ associated with the mean kinetic energy and $\epsilon$ associated with the turbulence kinetic energy,

$$
\begin{aligned}
\underline{\mathcal{E}} & =-\Upsilon_{i j} \frac{\partial \bar{U}_{i}}{\partial x_{j}}, \\
\epsilon & =-\Upsilon_{i j} \frac{\partial u_{i}^{\prime}}{\partial x_{j}}
\end{aligned}
$$

This set of equations is represented in a schematic form in figure 1.

In the limit case $\overline{\boldsymbol{U}}=\mathbf{0}$, the mean kinetic energy $\underline{E}$ and the mixed kinetic energy $\underline{e}$ vanish as do all energy exchange terms associated with these two quantities. The formulation reduces to the sole exchanges between turbulence kinetic energy and internal energy and describes the well-known rate of decay of turbulence kinetic energy and the paired gain of internal energy [5]. Similarly, the formulation reduces to the exchanges between mean kinetic energy and internal energy in the limit case $\boldsymbol{u}^{\prime}=\mathbf{0}$.

We compare the energy exchanges in the present formulation to the ternary decomposition of Chassaing [8]. While Chassaing [8] did not explicitly give the energy exchanges between the different parts of total energy, he gave enough information 


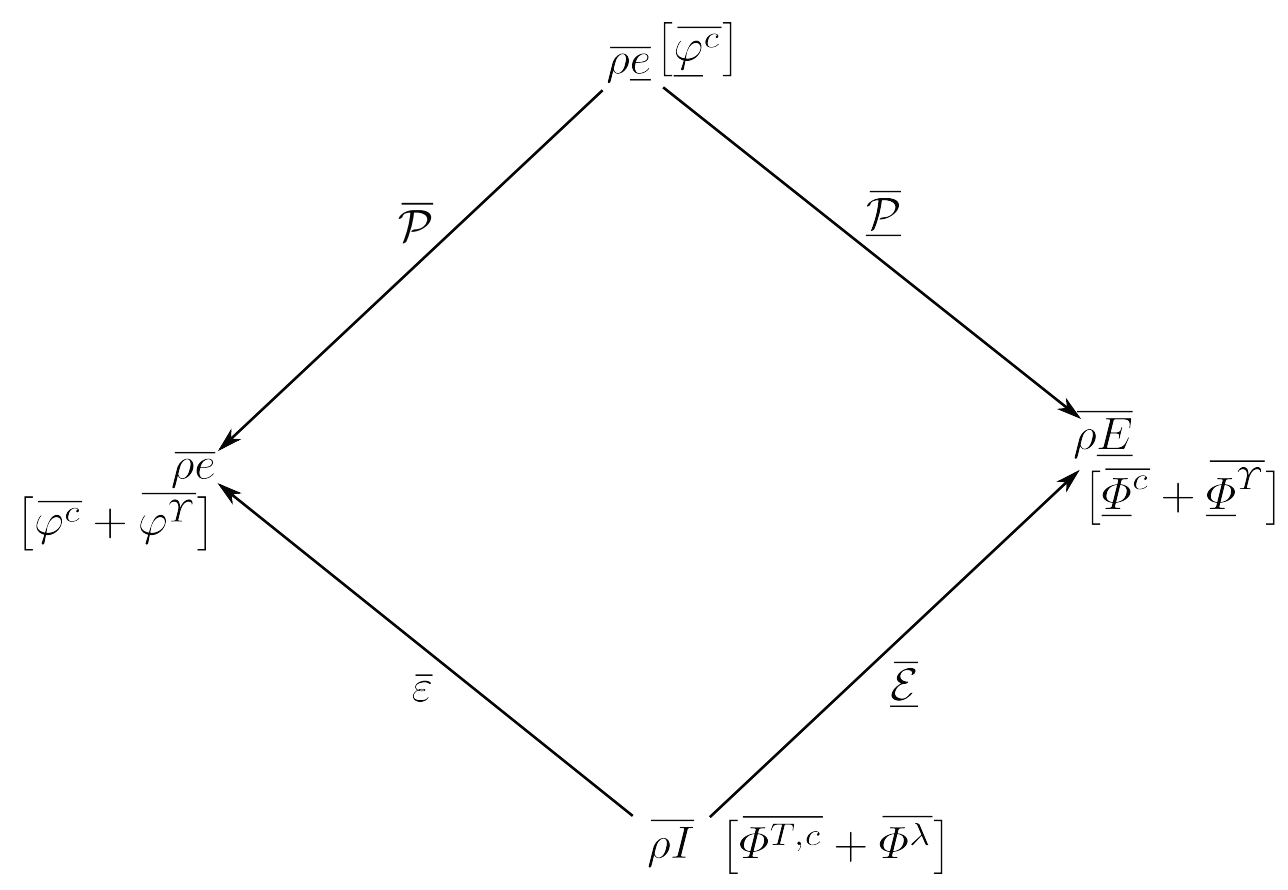

Figure 1 - Schematic representation of the energy exchanges between the four parts of total kinetic energy (in the one-stage formulation). An arrow represents an interaction between two quantities. The orientation of the arrow gives the sign of the term according to classical thermodynamic convention. (The term is positive in the right-hand side of the evolution equation of the quantity towards which the arrow points and negative in the other). The conservative energy transfers are represented within square brackets.

to identify the energy exchanges without ambiguity. The formulation of Chassaing [8] may be written using the notations of this paper as:

$$
\begin{aligned}
& \frac{\partial \overline{\rho \underline{E}}}{\partial t}=\underline{\Phi}^{c}+\overline{\Phi^{\Upsilon}}+\bar{Y}+(\overline{\mathcal{P}}+\bar{X}-\bar{Y})-\bar{X}+\overline{\mathcal{E}}, \\
& \frac{\partial \overline{\rho e}}{\partial t}=\overline{\varphi^{c}}+\overline{\varphi^{\Upsilon}}+(\overline{\mathcal{P}}-\bar{X})+\bar{X}+\bar{\epsilon} \\
& \frac{\partial \bar{\rho} \underline{\underline{e}}}{\partial t}=\overline{\varphi^{c}}-\bar{Y}-(\overline{\mathcal{P}}-\bar{X})-(\overline{\mathcal{P}}+\bar{X}-\bar{Y}), \\
& \frac{\partial \overline{\rho I}}{\partial t}=\overline{\Phi^{T, c}}+\overline{\Phi^{\lambda}}-\overline{\mathcal{E}}-\bar{\epsilon}
\end{aligned}
$$

with:

$$
\begin{aligned}
& \bar{X}=-\overline{\rho u_{i}^{\prime} u_{j}^{\prime}} \frac{\partial \bar{U}_{i}}{\partial x_{j}}, \\
& \bar{Y}=-\frac{\partial \overline{\rho u_{j}^{\prime}} \underline{\partial}}{\partial x_{j}} .
\end{aligned}
$$

It is represented in a schematic form in figure 2. 


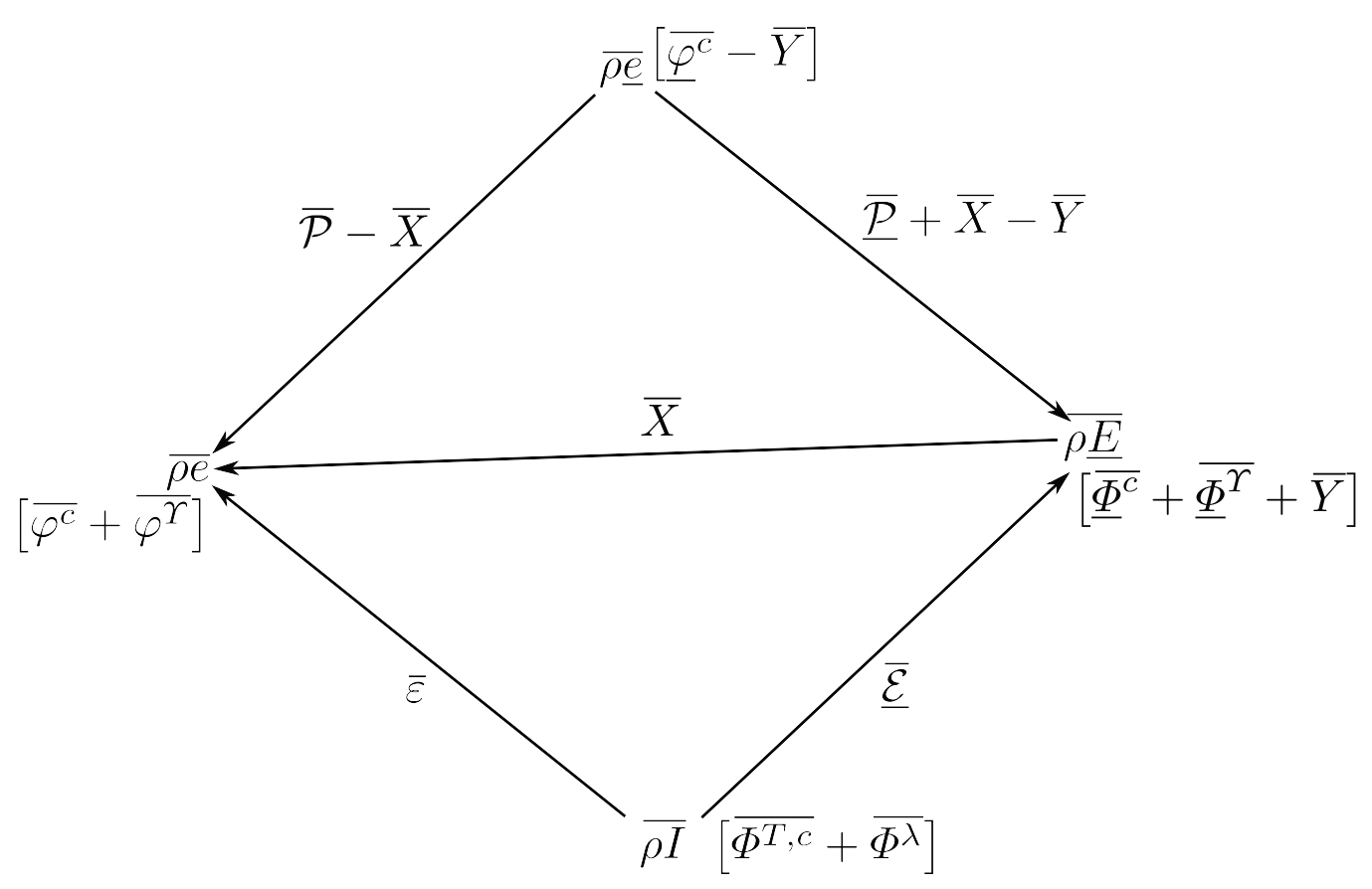

Figure 2 - Schematic representation of the energy exchanges between the four parts of total kinetic energy according to the formulation of Chassaing et al. [10]. Refer to the caption of figure 1 for some indications on how to read this diagram.

The formulation presented in this paper and the formulation of Chassaing [8] are mathematically equivalent. However, the energy exchanges identified and the interpretation given to the terms are different. The differences can be attributed to two main changes. First, the present formulation associates to the four parts of total energy a convective term related to both the mean and turbulent motion, that is of the transport by advection and diffusion. However, the formulation of Chassaing [8] only associates an advective term to the mixed kinetic energy $\bar{\rho} \underline{e}$, but no diffusive term. Due to this, the term $\bar{Y}$, diffusion of mixed kinetic energy in the present formulation, instead appears in the evolution equation of the mean kinetic energy $\bar{\rho} \underline{E}$ and is interpreted as the power of the Reynolds stress through the mean motion. In addition, there is in the formulation of Chassaing [8] a direct energy exchange $\bar{X}$ between turbulence kinetic energy and mean kinetic energy whereas any interaction between turbulence kinetic energy and mean kinetic energy occurs through the mixed kinetic energy in the present formulation. Both of these changes modify the energy exchange associated with mixed kinetic energy.

The formulation of Chassaing [8] is due to these two differences more similar to the classical incompressible representation of the energy exchanges, in which the term $\bar{Y}$ appears in the evolution equation of the mean kinetic energy and where there is a direct energy exchange between turbulence kinetic energy and mean kinetic energy. However, some elements suggest that the present formulation is more physical. Indeed, it associates a full convective term to each part of kinetic energy. We consider this as necessary as it is part of the material derivative. Besides, the 
formulation is more symmetrical with regard to the manner it deals with fluctuations and statistically averaged quantities. The formulation is not modified if the statistical average operator and the fluctuating part operator are substituted in equations (13) to $(22)$ and in the definitions of $\rho \underline{E}, \rho e$ and $\rho \underline{e}$. Finally, the formulation may be used to consider the instantaneous energy exchanges as it does not rely on simplifications only valid in the statistically averaged case. This ensures the consistency of the formulation, in the sense that the energy exchanges in both the instantaneous and statistically averaged cases are well-defined and are not conflicting. This consistency is important to give a physical interpretation to the energy exchange, as this lets us consider the statistically averaged energy exchanges as the statistical average of the associated instantaneous energy exchanges.

\subsection{Two-stage formulation}

The ternary decomposition of kinetic energy is taken further with the decomposition of density into a mean and fluctuating part $\rho=\bar{\rho}+\rho^{\prime}$. Namely, total energy $\rho(E+I)$ is decomposed into a mean density part $\bar{\rho}(E+I)$ and a fluctuating density part $\rho^{\prime}(E+I)$. Similarly, the mean kinetic energy $\rho \underline{E}$, the turbulence kinetic energy $\rho e$, the mixed kinetic energy $\rho \underline{e}$ and the internal energy per unit volume $\rho I$ are decomposed into a mean density part, $\bar{\rho} \underline{E}, \bar{\rho} e, \bar{\rho} \underline{e}$ and $\bar{\rho} I$ respectively, and a fluctuating density part, $\rho^{\prime} \underline{E}, \rho^{\prime} e, \rho^{\prime} \underline{e}$ and $\rho^{\prime} I$ respectively. This results in a eightfold decomposition of total energy.

In this eightfold decomposition of total energy, the terms of the ternary formulation are decomposed further in a mean and fluctuating density part. Any term $\alpha$ identified in the one-stage formulation is split into two terms in the two-stage formulation: $\alpha_{0}$, associated with the mean density part of total energy and $\alpha_{1}$, associated with the fluctuating density part of total energy. Moreover, additional terms appear that represent an interaction between the mean and fluctuating density part of total energy.

The energy exchanges between the eight parts of total energy may be written as:

$$
\begin{aligned}
& \frac{\partial \bar{\rho} \underline{\underline{E}}}{\partial t}=\overline{\Phi_{0}^{c}}+\overline{\Phi_{0}^{\Upsilon}}+\overline{\mathcal{P}_{0}}+\overline{Z^{c}}+\overline{Z^{\Upsilon}}+\overline{\mathcal{E}_{0}}, \\
& \frac{\partial \frac{\rho^{\prime} \underline{E}}{\partial t}}{\partial \overline{\Phi_{1}^{c}}}+\overline{\Phi_{1}^{\Upsilon}}+\overline{\mathcal{P}_{1}}-\overline{Z^{c}}-\overline{Z^{\Upsilon}}+\overline{\mathcal{E}_{1}}, \\
& \frac{\partial \bar{\rho} \bar{e}}{\partial t}=\overline{\varphi_{0}^{c}}+\overline{\varphi_{0}^{\Upsilon}}+\overline{\mathcal{P}_{0}}+\overline{\zeta^{c}}+\overline{\zeta^{\Upsilon}}+\overline{\epsilon_{0}}, \\
& \frac{\partial \overline{\rho^{\prime} e}}{\partial t}=\overline{\varphi_{1}^{c}}+\overline{\varphi_{1}^{\Upsilon}}+\overline{\mathcal{P}_{1}}-\overline{\zeta^{c}}-\overline{\zeta^{\Upsilon}}+\overline{\epsilon_{1}}, \\
& \frac{\partial \bar{\rho} \underline{\bar{e}}}{\partial t}=\overline{\varphi_{0}^{c}}-\overline{\mathcal{P}_{0}}-\overline{\mathcal{P}_{0}}+\overline{\zeta^{c}}, \\
& \frac{\partial \overline{\rho^{\prime} \underline{e}}}{\partial t}=\overline{\varphi^{c}}-\overline{\mathcal{P}_{1}}-\overline{\mathcal{P}_{1}}-\overline{\zeta^{c}}, \\
& \frac{\partial \bar{\rho} \bar{I}}{\partial t}=\overline{\Phi_{0}^{T, c}}+\overline{\Phi_{0}^{\lambda}}+\overline{Z^{T, c}}+\overline{Z^{\lambda}}-\overline{\mathcal{E}_{0}}-\overline{\epsilon_{0}},
\end{aligned}
$$




$$
\frac{\partial \overline{\rho^{\prime} I}}{\partial t}=\overline{\Phi_{1}^{T, c}}+\overline{\Phi_{1}^{\lambda}}-\overline{Z^{T, c}}-\overline{Z^{\lambda}}-\overline{\mathcal{E}_{1}}-\overline{\epsilon_{1}}
$$

with

$$
\begin{aligned}
& \underline{\Phi}_{0}^{c}=-\frac{\partial \bar{\rho} U_{j} \underline{E}}{\partial x_{j}}, \\
& \underline{\Phi}_{1}^{c}=-\frac{\partial \rho^{\prime} U_{j} \underline{E}}{\partial x_{j}}, \\
& \varphi_{0}^{c}=-\frac{\partial \bar{\rho} U_{j} e}{\partial x_{j}}, \\
& \varphi_{1}^{c}=-\frac{\partial \rho^{\prime} U_{j} e}{\partial x_{j}}, \\
& \underline{\varphi}_{0}^{c}=-\frac{\partial \bar{\rho} U_{j} \underline{e}}{\partial x_{j}}, \\
& \underline{\varphi}_{1}^{c}=-\frac{\partial \rho^{\prime} U_{j} \underline{e}}{\partial x_{j}}, \\
& \Phi_{0}^{T, c}=-\frac{\partial \bar{\rho} U_{j} I}{\partial x_{j}}, \\
& \Phi_{1}^{T, c}=-\frac{\partial \rho^{\prime} U_{j} I}{\partial x_{j}}, \\
& \underline{\Phi}_{0}^{\Upsilon}=\frac{\partial}{\partial x_{j}}\left(\frac{\Upsilon_{i j}}{\rho} \bar{\rho} \bar{U}_{i}\right), \\
& \underline{\Phi}_{1}^{\Upsilon}=\frac{\partial}{\partial x_{j}}\left(\frac{\Upsilon_{i j}}{\rho} \rho^{\prime} \bar{U}_{i}\right), \\
& \varphi_{0}^{\Upsilon}=\frac{\partial}{\partial x_{j}}\left(\frac{\Upsilon_{i j}}{\rho} \bar{\rho} u_{i}^{\prime}\right), \\
& \varphi_{1}^{\Upsilon}=\frac{\partial}{\partial x_{j}}\left(\frac{\Upsilon_{i j}}{\rho} \rho^{\prime} u_{i}^{\prime}\right), \\
& \Phi_{0}^{\lambda}=\frac{\partial}{\partial x_{j}}\left(\frac{\bar{\rho}}{\rho} \lambda \frac{\partial T}{\partial x_{j}}\right), \\
& \Phi_{1}^{\lambda}=\frac{\partial}{\partial x_{j}}\left(\frac{\rho^{\prime}}{\rho} \lambda \frac{\partial T}{\partial x_{j}}\right), \\
& \mathcal{P}_{0}=-\bar{\rho} u_{i}^{\prime} U_{j} \frac{\partial \bar{U}_{i}}{\partial x_{j}}+\bar{\rho} u_{i}^{\prime} \overline{U_{j} \frac{\partial u_{i}^{\prime}}{\partial x_{j}}}-\bar{\rho} u_{i}^{\prime} \frac{\overline{1} \frac{\partial \Upsilon_{i j}}{\partial x_{j}}}{,}, \\
& \mathcal{P}_{1}=-\rho^{\prime} u_{i}^{\prime} U_{j} \frac{\partial \bar{U}_{i}}{\partial x_{j}}+\rho^{\prime} u_{i}^{\prime} \overline{U_{j} \frac{\partial u_{i}^{\prime}}{\partial x_{j}}}-\rho^{\prime} u_{i}^{\prime} \frac{\overline{1} \frac{\partial \Upsilon_{i j}}{\partial x_{j}}}{,} \\
& \underline{\mathcal{P}}_{0}=-\bar{\rho} \bar{U}_{i} U_{j} \frac{\partial u_{i}^{\prime}}{\partial x_{j}}+\bar{\rho} \bar{U}_{i}\left(U_{j} \frac{\partial U_{i}}{\partial x_{j}}\right)^{\prime}-\bar{\rho} \bar{U}_{i}\left(\frac{1}{\rho} \frac{\partial \Upsilon_{i j}}{\partial x_{j}}\right)^{\prime}, \\
& \underline{\mathcal{P}}_{1}=-\rho^{\prime} \bar{U}_{i} U_{j} \frac{\partial u_{i}^{\prime}}{\partial x_{j}}+\rho^{\prime} \bar{U}_{i}\left(U_{j} \frac{\partial U_{i}}{\partial x_{j}}\right)^{\prime}-\rho^{\prime} \bar{U}_{i}\left(\frac{1}{\rho} \frac{\partial \Upsilon_{i j}}{\partial x_{j}}\right)^{\prime}, \\
& \underline{\mathcal{E}}_{0}=-\frac{\bar{\rho} \Upsilon_{i j}}{\rho} \frac{\partial \bar{U}_{i}}{\partial x_{j}} \\
& \underline{\mathcal{E}}_{1}=-\frac{\rho^{\prime} \Upsilon_{i j}}{\rho} \frac{\partial \bar{U}_{i}}{\partial x_{j}} \\
& \epsilon_{0}=-\frac{\bar{\rho} \Upsilon_{i j}}{\rho} \frac{\partial u_{i}^{\prime}}{\partial x_{j}} \\
& \epsilon_{1}=-\frac{\rho^{\prime} \Upsilon_{i j}}{\rho} \frac{\partial u_{i}^{\prime}}{\partial x_{j}}
\end{aligned}
$$

and where we identify the following terms:

- the energy dilatation correlation $Z^{c}$,

$$
Z^{c}=(E+I) \frac{\partial \bar{\rho} U_{j}-\overline{\rho U_{j}}}{\partial x_{j}},
$$

decomposed into: $\underline{Z}^{c}$ associated with the mean kinetic energy, $\zeta^{c}$ associated with the turbulence kinetic energy, $\underline{\zeta}^{c}$ associated with the mixed kinetic energy and $Z^{T, c}$ associated with the internal energy,

$$
\underline{Z}^{c}=\underline{E} \frac{\partial \bar{\rho} U_{j}-\overline{\rho U_{j}}}{\partial x_{j}}
$$




$$
\begin{aligned}
\zeta^{c} & =e \frac{\partial \bar{\rho} U_{j}-\overline{\rho U_{j}}}{\partial x_{j}}, \\
\underline{\zeta}^{c} & =\underline{e} \frac{\partial \bar{\rho} U_{j}-\overline{\rho U_{j}}}{\partial x_{j}}, \\
Z^{T, c} & =I \frac{\partial \bar{\rho} U_{j}-\overline{\rho U_{j}}}{\partial x_{j}},
\end{aligned}
$$

- the interaction between the mean and fluctuating density part of total energy by the total stress $Z^{\Upsilon}$,

$$
Z^{\Upsilon}=-\Upsilon_{i j} U_{i} \frac{\partial}{\partial x_{j}}\left(\frac{\bar{\rho}}{\rho}\right)
$$

decomposed into: $\underline{Z}^{\Upsilon}$ associated with the mean kinetic energy and $\zeta^{\Upsilon}$ associated with the turbulence kinetic energy,

$$
\begin{aligned}
\underline{Z}^{\Upsilon} & =-\Upsilon_{i j} \bar{U}_{i} \frac{\partial}{\partial x_{j}}\left(\frac{\bar{\rho}}{\rho}\right), \\
\zeta^{\Upsilon} & =-\Upsilon_{i j} u_{i}^{\prime} \frac{\partial}{\partial x_{j}}\left(\frac{\bar{\rho}}{\rho}\right),
\end{aligned}
$$

- the interaction between the mean and fluctuating density part of total energy by conduction $Z^{\lambda}$.

$$
Z^{\lambda}=-\lambda \frac{\partial T}{\partial x_{j}} \frac{\partial}{\partial x_{j}}\left(\frac{\bar{\rho}}{\rho}\right)
$$

This set of equations is represented in a schematic form in figure 3.

The two-stage formulation includes the mean density turbulence kinetic energy. In the remaining part of the paper, we will establish its evolution equation in the spectral domain.

\section{Spectral equation of the mean density turbulence kinetic energy}

The investigation of the mean density turbulence kinetic energy evolution equation provides information on the energy exchanges associated with this quantity in the spatial direction. The study may be extended to the spectral domain by establishing its spectral evolution equation. This investigation permits to give the effect of the energy exchanges with regard to the size of the turbulent structures.

In order to write the spectral equation, we need to consider a flow with at least one direction of homogeneity. Without loss of generality, we consider a turbulent flow with two homogeneous and periodic directions, $x$ and $z$. The inhomogeneous direction is denoted $y$. The dimensions of the domain in the $x, y$ and $z$ directions are denoted $L_{x}, L_{y}$ and $L_{z}$ respectively. Since the flow is periodic in the $x$ and $z$ 


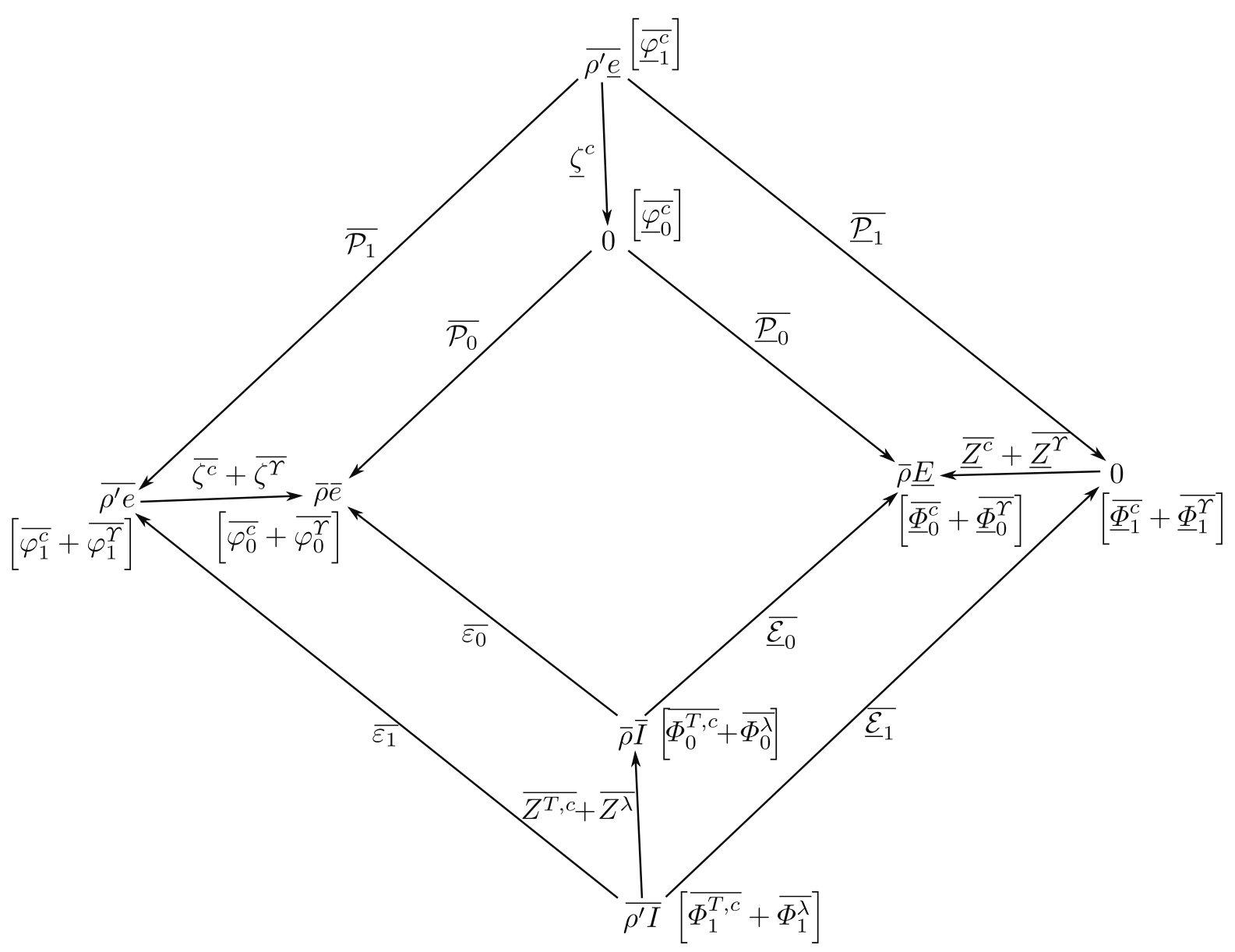

Figure 3 - Schematic representation of the energy exchanges between the eight parts of total kinetic energy (in the two-stage formulation). Refer to the caption of figure 1 for some indications on how to read this diagram.

directions, we perform the Fourier transform in the $x$ and $z$ directions only and leave the $y$ direction untransformed. Any physical quantity $g(x, y, z)$ can be expressed as a Fourier series,

$$
g(\boldsymbol{x}, y, t)=\sum_{p, q=-\infty}^{\infty} \widehat{g}\left(\boldsymbol{k}_{p, q}, y, t\right) \mathrm{e}^{\mathrm{i} \boldsymbol{k}_{p, q} \cdot \boldsymbol{x}},
$$

where $p$ and $q$ are positive or negative integers, $\boldsymbol{x}=(x, z)$ is the position vector in the $x O z$ plane and $\boldsymbol{k}_{p, q}=\boldsymbol{k}=\left(k_{x}, k_{z}\right)=\left(\frac{2 \pi p}{L_{x}}, \frac{2 \pi q}{L_{z}}\right)$ is the position vector in the $k_{x} O k_{z}$ plane. The Fourier coefficients of the Fourier series expansion of $g$ are denoted with the hat operator $(\widehat{)})$ and are given by [33]:

$$
\widehat{g}(\boldsymbol{k}, y, t)=\frac{1}{L_{x} L_{z}} \int_{0}^{L_{x}} \int_{0}^{L_{z}} g(\boldsymbol{x}, y, t) \mathrm{e}^{-\mathrm{i} \boldsymbol{k} \cdot \boldsymbol{x}} d \boldsymbol{x} .
$$

The half-trace of the velocity fluctuation correlation tensor $\bar{e}$ is equal to half the correlation

$$
C(\boldsymbol{r}, y, t)=\overline{u_{i}^{\prime}(\boldsymbol{r}, y, t) u_{i}^{\prime}(\boldsymbol{x}+\boldsymbol{r}, y, t)}
$$


in the particular case $\boldsymbol{r}=0$, that is $\bar{e}(y, t)=\frac{1}{2} C(\mathbf{0}, y, t)$. We can express $C$ as a Fourier series from (46). The Fourier coefficients $\widehat{C}$ can be written as [33, 42]

$$
\widehat{C}(\boldsymbol{k}, y, t)=\widehat{{\widehat{u_{i}^{\prime}}}^{*}(\boldsymbol{k}, y, t) \widehat{u_{i}^{\prime}}(\boldsymbol{k}, y, t)}
$$

where the asterisk $\left(^{*}\right)$ denotes the complex conjugate. Henceforth, we denote $\bar{e}$ and call spectral equivalent of $\bar{e}$ the half of the spectral correlation $\widehat{C}$,

$$
\bar{e}=\frac{1}{2} \widehat{u_{i}^{\prime} * \widehat{u_{i}^{\prime}}} \text {. }
$$

From similar arguments, we shall associate to each term of the evolution equation of the half-trace of the velocity fluctuation correlation tensor a spectral equivalent.

Spectral equations of the turbulence kinetic energy were given by Domaradzki et al. [19], Marati et al. [36], Dunn and Morrison [20], Bolotnov et al. [7], Lee and Moser [31], Mizuno [38] in the incompressible case and Aulery et al. [1] in the variable density case. The present decomposition gives a clear one-to-one correspondence between the terms of the spectral and spatial decompositions. To this end, a purely spectral term with no spatial contribution has to be considered. The decomposition is similar to the decomposition of Mizuno [38] at the incompressible limit.

The spectral evolution equation of the mean density turbulence kinetic energy may be written as:

$$
\frac{\partial \bar{e}}{\partial t}=\overline{\check{\varphi}_{0}^{c}}+\overline{\check{\varphi}_{0}^{\Upsilon}}+\overline{\check{\mathcal{P}}_{0}}+\check{\zeta}^{c}+\overline{\check{\zeta}^{\Upsilon}}+\check{\epsilon}_{0}
$$

where we identify the following terms:

- the convection $\overline{\breve{\varphi}_{0}^{c}}$, associated with the spatial convection $\overline{\varphi_{0}^{c}}$,

$$
\overline{\check{\varphi}_{0}^{c}}=-\operatorname{Re}\left(-\frac{1}{2} \frac{\partial \bar{\rho} \widehat{\widehat{u}_{i}^{*}{\widehat{u_{i}^{\prime} U_{j}}}^{2}}}{\partial x_{j}}\right),
$$

- the transfer by the total stress $\overline{\check{\varphi}_{0}^{\Upsilon}}$, associated with the spatial transfer by the total stress $\overline{\varphi_{0}^{\Upsilon}}$,

$$
\overline{\check{\varphi}_{0}^{\Upsilon}}=\operatorname{Re}\left(\frac{\partial}{\partial x_{j}} \bar{\rho} \widehat{\overline{u_{i}^{\prime}} * \widehat{\Upsilon_{i j}}}\right),
$$

- the interaction with mixed kinetic energy $\check{\mathcal{P}}_{0}$, associated with the spatial interaction with mixed kinetic energy $\overline{\mathcal{P}_{0}}$,

$$
\overline{\mathcal{P}_{0}}=\operatorname{Re}\left(-\bar{\rho}{\widehat{u_{i}^{\prime}}}^{*} \widehat{u}_{j}^{\prime} \frac{\partial \bar{U}_{i}}{\partial x_{j}}\right),
$$

- the kinetic energy dilation correlation $\check{\zeta}^{c}$, associated with the spatial kinetic energy dilation correlation $\overline{\zeta^{c}}$,

$$
\overline{\check{\zeta}^{c}}=\operatorname{Re}\left(\frac{1}{2} \widehat{\widehat{u}_{i}^{\prime}{ }^{*}} \overline{u_{i}^{\prime} \overline{\frac{\partial U_{j}-\bar{\rho} U_{j}}{\partial x_{j}}}}\right),
$$


- the interaction with fluctuating density kinetic energy by the total stress $\bar{\zeta}$, associated with the spatial interaction with fluctuating density kinetic energy by the total stress $\overline{\zeta^{\Upsilon}}$,

$$
\overline{\check{\zeta}^{\Upsilon}}=\operatorname{Re}\left(\overline{{\widehat{u_{i}^{\prime}}}^{*} \overline{\Upsilon_{i j} \frac{\partial}{\partial x_{j}}\left(\frac{\bar{\rho}}{\rho}\right)}}\right),
$$

- the interaction between kinetic energy and internal energy $\overline{\epsilon_{0}}$, associated with the spatial interaction with internal energy $\overline{\epsilon_{0}}$,

$$
\bar{\epsilon}_{0}=\operatorname{Re}\left(\overline{-\bar{\rho}} \overline{\frac{\partial u_{i}^{\prime}}{\partial x_{j}}} \frac{\widehat{\Upsilon}_{i j}}{\rho}\right)
$$

- the purely spectral term $\check{\Xi}$, with no contribution in the spatial domain,

$$
\check{\Xi}=\operatorname{Re}\left(\frac{1}{2} \widehat{\frac{\partial \bar{\rho} u_{i}^{\prime}}{\partial x_{j}}}{\widehat{u_{i}^{\prime} u_{j}^{\prime}}}^{*}-\frac{1}{2} \widehat{u_{i}^{\prime *}} \widehat{\widehat{u_{j}^{\prime} \frac{\partial \bar{\rho} u_{i}^{\prime}}{\partial x_{j}}}}\right),
$$

where Re denotes the real part operator.

The terms are decomposed in order to have a one-to-one correspondence with the terms of the spatial decomposition. The inverted hat operator $\left({ }^{\sim}\right)$ is used to indicate the spectral equivalent of a spatial term. The spectral and spatial terms are tied closely. Given a spectral term written in the form $\operatorname{Re}\left(\overline{\widehat{u}_{i}^{*}} \widehat{a}\right)$, for any $a$, the associated spatial term is $\overline{u_{i}^{\prime} a}$. The spectral term comes from the Fourier coefficients of the spatial two-point correlation between $u_{i}^{\prime}$ and $a[33]$.

The purely spectral term $\stackrel{\bar{\Xi}}{\Xi}$ has no associated spatial term. The summation over the whole wavenumber space of its spectra is zero. In other words, this term has no effect on the spatial balance of kinetic energy but contributes to the interscale redistribution of kinetic energy.

\section{Conclusion}

The ternary decomposition of kinetic energy gives another angle of approach to the study of energy exchanges in turbulent flows. Based on the classical Reynolds averaging, the decomposition leads to the definition of a turbulence kinetic energy, a mean kinetic energy and a mixed kinetic energy. This new term, specific to the formulation, is related to the interaction between the turbulent motion and the mean motion. In the formulation, any energy exchange between the turbulence kinetic energy and the mean kinetic energy goes through the mixed term, which adds its contribution to the exchange. The formulation is decomposed further in order to include the mean density turbulence kinetic energy, product of the mean 
density and the half-trace of the velocity fluctuation correlation tensor. This is done by splitting the density in a mean part and a fluctuating part. Contrary to the turbulence kinetic energy, the equation of the mean density turbulence kinetic energy can easily be extended to the spectral domain. This associates a spectral equivalent to each spatial term and adds a purely spectral term to the decomposition.

\section{References}

[1] F. Aulery, A. Toutant, F. Bataille, and Y. Zhou. Energy transfer process of anisothermal wall-bounded flows. Physics Letters A, 379(24):1520-1526, 2015.

[2] F. Aulery, D. Dupuy, A. Toutant, F. Bataille, and Y. Zhou. Spectral analysis of turbulence in anisothermal channel flows. Computers \& Fluids, 151:115-131, 2017.

[3] B. Aupoix. Introduction to turbulence modelling for compressible flows. VKI lecture series, 4:H1-H64, 2000.

[4] F. Bataille, R. Rubinstein, and M. Y. Hussaini. Eddy viscosity and diffusivity modeling. Physics Letters A, 346(1):168-173, 2005.

[5] G. K. Batchelor. The theory of homogeneous turbulence. Cambridge university press, 1953.

[6] P. T. Bauer, G. W. Zumwalt, and L. J. Fila. A numerical method and an extension of the korst jet mixing theory for multispecie turbulent jet mixing. AIAA paper 68 (112). In 6th Aerospace Sciences Meeting, New York, 1968.

[7] I. A. Bolotnov, R. T. Lahey, D. A. Drew, K. E. Jansen, and A. A. Oberai. Spectral analysis of turbulence based on the DNS of a channel flow. Computers \& Fluids, 39 (4):640-655, 2010.

[8] P. Chassaing. Une alternative à la formulation des équations du mouvement turbulent d'un fluide à masse volumique variable. Journal de mécanique théorique et appliquée, 4(3):375-389, 1985.

[9] P. Chassaing. The modeling of variable density turbulent flows. a review of first-order closure schemes. Flow, Turbulence and Combustion, 66(4):293-332, 2001.

[10] P. Chassaing, R. A. Antonia, F. Anselmet, L. Joly, and S. Sarkar. Variable density fluid turbulence. Springer Science \& Business Media, 2013.

[11] A. Cimarelli, E. De Angelis, and C. M. Casciola. Paths of energy in turbulent channel flows. J. Fluid Mech., 715:436-451, 2013.

[12] A. Cimarelli, G. Cocconi, B. Frohnapfel, and E. De Angelis. Spectral enstrophy budget in a shear-less flow with turbulent/non-turbulent interface. Phys. Fluids, 27 (12):125106, 2015.

[13] A. Cimarelli, E. De Angelis, P. Schlatter, G. Brethouwer, A. Talamelli, and C. M. Casciola. Sources and fluxes of scale energy in the overlap layer of wall turbulence. $J$. Fluid Mech., 771:407-423, 2015.

[14] A. Cimarelli, E. De Angelis, J. Jiménez, and C. M. Casciola. Cascades and wallnormal fluxes in turbulent channel flows. J. Fluid Mech., 796:417-436, 2016. ISSN 1469-7645.

[15] A. W. Cook and Y. Zhou. Energy transfer in Rayleigh-Taylor instability. Phys. Rev. E, 66:026312, 2002.

[16] J. Cousteix and B. Aupoix. Turbulence models for compressible flows. In Special Course Three-Dimensional Supersonic and Hypersonic Flows Including Separation. AGARD/FDP-VKI Special course, 1989. 
[17] X. Daguenet-Frick, A. Toutant, F. Bataille, and G. Olalde. Numerical investigation of a ceramic high-temperature pressurized-air solar receiver. Solar Energy, 90(0): 164-178, 2013. ISSN 0038-092X.

[18] J. A. Domaradzki and R. S. Rogallo. Local energy transfer and nonlocal interactions in homogeneous, isotropic turbulence. Physics of Fluids A: Fluid Dynamics, 2(3): 413-426, 1990.

[19] J. A. Domaradzki, W. Liu, C. Hartel, and L. Kleiser. Energy transfer in numerically simulated wall-bounded turbulent flows. Phys. Fluids, 6(4):1583-1599, 1994.

[20] D. C. Dunn and J. F. Morrison. Analysis of the energy budget in turbulent channel flow using orthogonal wavelets. Computers \& Fluids, 34(2):199-224, 2005.

[21] A. Favre. The equations of compressible turbulent gases. Technical Report AD0622097, DTIC Document, 1965.

[22] A. Favre. Statistical equations of turbulent gases. Problems of Hydrodynamics and Continuum Mechanics, pages 231-266, 1969.

[23] A. Favre. Turbulence: Space-time statistical properties and behavior in supersonic flows. Phys. Fluids, 26(10):2851-2863, 1983.

[24] E. Garnier, N. Adams, and P. Sagaut. Large eddy simulation for compressible flows. Springer Science \& Business Media, 2009.

[25] T. B. Gatski and J. P. Bonnet. Compressibility, turbulence and high speed flow. Academic Press, 2013.

[26] S. E. Guarini, R. D. Moser, K. Shariff, and A. Wray. Direct numerical simulation of a supersonic turbulent boundary layer at Mach 2.5. J. Fluid Mech., 414:1-33, 2000.

[27] H. Ha Minh, B. E. Launder, and J. MacInnes. A new approach to the analysis of turbulent mixing in variable density flows. In 3rd Turbulent Shear Flow Symp. p, volume 19, 1981.

[28] H. Ha Minh, B. E. Launder, and J. MacInnes. The turbulence modelling of variable density flows-a mixed-weighted decomposition. In Turbulent Shear Flows 3, pages 291-308. Springer, 1982.

[29] P. G. Huang, G. N. Coleman, and P. Bradshaw. Compressible turbulent channel flows: DNS results and modelling. J. Fluid Mech., 305:185-218, 1995.

[30] S. Kida and S. A. Orszag. Energy and spectral dynamics in decaying compressible turbulence. Journal of Scientific Computing, 7(1):1-34, 1992. ISSN 0885-7474.

[31] M. Lee and R. D. Moser. Spectral analysis on reynolds stress transport equation in high firewall-bounded turbulence. In International Symposium on Turbulence and Shear Flow Phenomena (TSFP-9), Melbourne, pages 4A-3, 2006.

[32] S. K. Lele. Compressibility effects on turbulence. Annu. Rev. Fluid Mech., 26(1): 211-254, 1994.

[33] M. Lesieur. Turbulence in Fluids. Fluid Mechanics and Its Applications. Springer, 2008. ISBN 9781402064357.

[34] J. L. Lumley. Spectral energy budget in wall turbulence. Phys. Fluids, 7(2):190-196, 1964.

[35] T. Maeder, N. A. Adams, and L. Kleiser. Direct simulation of turbulent supersonic boundary layers by an extended temporal approach. J. Fluid Mech., 429:187-216, 2001.

[36] N Marati, CM Casciola, and R Piva. Energy cascade and spatial fluxes in wall turbulence. J. Fluid Mech., 521:191-215, 2004.

[37] L. Mathelin, F. Bataille, and Y. Zhou. Theoretical investigation of some thermal effects in turbulence modeling. Theoretical and Computational Fluid Dynamics, 22 (6):471-483, 2008. 
[38] Y. Mizuno. Spectra of energy transport in turbulent channel flows for moderate reynolds numbers. J. Fluid Mech., 805:171-187, 2016.

[39] M. V. Morkovin. The Mechanics of Turbulence. Gordon and Breach, 1964.

[40] F. Nicoud. Conservative high-order finite-difference schemes for low-Mach number flows. J. Comput. Phys., 158(1):71-97, 2000.

[41] S. Pirozzoli, F. Grasso, and T. B. Gatski. Direct numerical simulation and analysis of a spatially evolving supersonic turbulent boundary layer at $\mathrm{M}=2.25$. Phys. Fluids, 16(3):530-545, 2004.

[42] S. B. Pope. Turbulent Flows. Cambridge University Press, 2000.

[43] O. Reynolds. On the dynamical theory of incompressible viscous fluids and the determination of the criterion. Proceedings of the Royal Society of London, 56(336-339): 40-45, 1894.

[44] A. J. L. Rodriguez and G. Emanuel. Compressible rotational flows generated by the substitution principle. Phys. Fluids, 31:1058-1063, 1988.

[45] S. Serra, A. Toutant, and F. Bataille. Thermal large eddy simulation in a very simplified geometry of a solar receiver. Heat Transfer Engineering, 33(6):505-524, 2012.

[46] S. Serra, A. Toutant, F. Bataille, and Y. Zhou. High-temperature gradient effect on a turbulent channel flow using thermal large-eddy simulation in physical and spectral spaces. J. Turbulence, 13:N49, 2012.

[47] S. Serra, A. Toutant, F. Bataille, and Y. Zhou. Turbulent kinetic energy spectrum in very anisothermal flows. Physics Letters A, 376(45):3177-3184, 2012.

[48] G. S. Sidharth, A. Kartha, and G. V. Candler. Filtered velocity based LES of mixing in high speed recirculating shear flow. In 46th AIAA Fluid Dynamics Conference, page 3184, 2016.

[49] A. Toutant and F. Bataille. Turbulence statistics in a fully developed channel flow submitted to a high temperature gradient. International Journal of Thermal Sciences, 74:104-118, 2013.

[50] A. Toutant, E. Labourasse, O. Lebaigue, and O. Simonin. DNS of the interaction between a deformable buoyant bubble and a spatially decaying turbulence: a priori tests for LES two-phase flow modelling. Computers \& Fluids, 37(7):877-886, 2008.

[51] D. C. Wilcox. Turbulence modeling for CFD, volume 2. DCW industries La Canada, CA, 1998.

[52] C.-S. Yih. Exact solutions for steady two-dimensional flow of a stratified fluid. $J$. Fluid Mech., 9(02):161-174, 1960.

[53] Y. Zhou. Degrees of locality of energy transfer in the inertial range. Physics of Fluids A: Fluid Dynamics, 5(5):1092-1094, 1993.

[54] Y. Zhou. Interacting scales and energy transfer in isotropic turbulence. Physics of Fluids A: Fluid Dynamics, 5(10):2511-2524, 1993. 